\title{
CHARACTERIZATIONS OF INNER PRODUCT SPACES BY MEANS OF NORM ONE POINTS
}

\author{
JOSÉ MENDOZA and TIJANI PAKHROU*
}

\begin{abstract}
Let $X$ be a a real normed linear space of dimension at least three, with unit sphere $S_{X}$. In this paper we prove that $X$ is an inner product space if and only if every three point subset of $S_{X}$ has a Chebyshev center in its convex hull. We also give other characterizations expressed in terms of centers of three point subsets of $S_{X}$ only. We use in these characterizations Chebyshev centers as well as Fermat centers and $p$-centers.
\end{abstract}

\section{Introduction}

Let $(X,\|\cdot\|)$ be a real normed linear space with unit sphere $S_{X}$. Let $x_{0}$ be an element of $X$ and let $A$ be a non-empty bounded subset of $X$. We write

$$
r\left(x_{0}, A\right)=\sup \left\{\left\|y-x_{0}\right\|: y \in A\right\}
$$

and

$$
r(A)=\inf \{r(x, A): x \in X\} .
$$

The number $r(A)$ is called the Chebyshev radius of $A$, and we write

$$
Z(A)=\{x \in X: r(x, A)=r(A)\} .
$$

This set (possibly empty) is known as the Chebyshev center set of $A$. Any point of $Z(A)$ is said to be a Chebyshev center of $A$. Note that "Chebyshev center" denotes a set as well as any point in that set. This should not cause any confusion.

Given $r>0$ we denote by $B\left(x_{0}, r\right)$ the closed ball centered at $x_{0}$ with radius $r$, that is,

$$
B\left(x_{0}, r\right)=\left\{x \in X:\left\|x-x_{0}\right\| \leq r\right\} .
$$

Notice that if $x_{0}$ is a Chebyshev center of $A$ and we take $r=r(A)$, then

$$
A \subset B\left(x_{0}, r\right) .
$$

\footnotetext{
* Supported in part by D.G.I.C.Y.T. grant BFM 2001-1284.

Received January 20, 2004; in revised form May 24, 2004.
} 
In fact, the Chebyshev radius of $A, r(A)$, is the smallest number $r \geq 0$ for which there exists $x \in X$ such that

$$
A \subset B(x, r) .
$$

In [1] Amir gave the following condition as a characterization of inner product spaces (IPS in short) among real normed linear spaces of dimension at least three (we keep the number assigned in the book):

(15.14) If $a_{1}, a_{2}, a_{3}$ are norm one points in $X$ such that $r\left(\left\{a_{1}, a_{2}, a_{3}\right\}\right)=1$, then $\mathbf{0}$ is in the convex hull of $\left\{a_{1}, a_{2}, a_{3}\right\}$.

Since $a_{1}, a_{2}, a_{3}$ are norm one points, $r\left(\left\{a_{1}, a_{2}, a_{3}\right\}\right)=1$ just means that $\mathbf{0}$ is Chebyshev center of $\left\{a_{1}, a_{2}, a_{3}\right\}$. Therefore, we can reformulate (15.14) as follows:

(15.14') If $a_{1}, a_{2}, a_{3}$ are norm one points in $X$ and $\mathbf{0}$ is a Chebyshev center of the set $\left\{a_{1}, a_{2}, a_{3}\right\}$, then $\mathbf{0}$ is in its convex hull.

However, we have shown in [8] that there is a mistake in Amir's book: condition (15.14) (or (15.14')) does not characterize IPS.

At this point a natural question arises: How must Amir's condition be modified to keep its spirit and get a right characterization?

On one hand, Amir's condition is motivated by the Garkavi-Klee theorem (see [6], [7] or (15.1) and (15.2) of [1]):

Theorem 1 (Garkavi-Klee). Let $X$ be a real normed linear space of dimension at least three. Then $X$ is an IPS if and only if the following condition holds:

(GK) Every three point subset of X has a Chebyshev center in its convex hull.

On the other hand, one of the main features of equivalent conditions (15.14) and (15.14') is that they are expressed only in terms of norm one points.

With this in mind, we have been looking for conditions involving Chebyshev centers of sets of three norm one points. That is, we have dealt with Chebyshev centers of triangles whose vertices are norm one points, or in other words, triangles inscribed in the unit sphere. We have found two conditions. The first one is just (15.14') with an additional requirement, and the second one is just condition (GK), writing $S_{X}$ instead of $X$. They are the following:

(A) If $a_{1}, a_{2}, a_{3}$ are norm one points in $X$ then $Z\left(\left\{a_{1}, a_{2}, a_{3}\right\}\right)$ is nonempty, and if $\mathbf{0}$ is a Chebyshev center of the set $\left\{a_{1}, a_{2}, a_{3}\right\}$, then $\mathbf{0}$ is in its convex hull. 
$\left(\mathrm{GK}^{s}\right)$ Every three point subset of $S_{X}$ has a Chebyshev center in its convex hull.

In section 1 (Theorem 1) we show that they are indeed characterizations of IPS.

Since Chebyshev centers are just particular kinds of centers, it is natural to consider the preceding conditions for another kinds, too. Let us see this.

Take $p \geq 1$. Given a three point set $\Delta=\left\{a_{1}, a_{2}, a_{3}\right\}$ in $X$, we consider the function on $X$,

$$
x \mapsto r_{p}(x, \Delta)=\left(\sum_{i=1}^{3}\left\|a_{i}-x\right\|^{p}\right)^{1 / p} .
$$

Write

$$
Z^{p}(\Delta)=\left\{z \in X: r_{p}(z, \Delta)=\inf _{x \in X} r_{p}(x, \Delta)\right\} .
$$

This set (perhaps empty) is the set of $p$-centers of $\Delta$. In the case $p=1$ the $p$ centers are called Fermat centers or Fermat-Torricelli medians of the triangle $\Delta$. In the case $p=2$ they are sometimes called barycenters.

We consider now conditions analogous to (A) and $\left(\mathrm{GK}^{s}\right)$ for these centers.

( $\left.\mathrm{A}_{p}\right)$ If $a_{1}, a_{2}, a_{3}$ are norm one points in $X$ then $Z^{p}\left(\left\{a_{1}, a_{2}, a_{3}\right\}\right)$ is nonempty, and if $\mathbf{0}$ is a p-center of the set $\left\{a_{1}, a_{2}, a_{3}\right\}$, then $\mathbf{0}$ is in its convex hull.

$\left(\mathrm{GK}_{p}^{s}\right)$ Every three point subset of $S_{X}$ has a p-center in its convex hull.

At this point, the following question arises: Do these conditions characterize IPS (among real normed linear spaces of dimension at least three)?

We devote the other two sections of this paper (sections 2 and 3) to this question. We show that in the case $p=1$, that is, for Fermat centers, the answer is affirmative (section 2). Concerning the case $p>1$, we prove that the answer is also affirmative for condition $\left(\mathrm{GK}_{p}^{s}\right)$ (section 3), but we have not been able to get an answer for condition $\left(\mathrm{A}_{p}\right)$.

While section 1 based on the Garkavi-Klee theorem, the main tool in sections 2 and 3 is a theorem recently proved by Benítez, Fernández and Soriano. It is the exact analogue to the Garkavi-Klee theorem, with $p$-centers instead of Chebyshev centers. The case $p>1$ was proved in [2], [3], and the case $p=1$, in [4]. This is the result:

Theorem 2 (Benítez-Fernández-Soriano). Let $X$ be a real normed linear space of dimension at least three, and let $p \geq 1$. Then $X$ is an IPS if and only if the following condition holds:

$\left(\mathrm{GK}_{p}\right)$ Every three point subset of $X$ has a p-center in its convex hull. 
REMARK 1. One should notice that with the usual conventions, $p$-centers for $p=+\infty$ are just Chebyshev centers. Thus, $\left(\mathrm{A}_{\infty}\right)$ would coincide with $(\mathrm{A})$, and $\left(\mathrm{GK}_{\infty}^{s}\right)$, with $\mathrm{GK}^{s}$. With this convention, the preceding theorem holds even for $p=+\infty$ : in this case, it is just the Garkavi-Klee theorem.

Given a set $B$, we will denote by $\operatorname{conv}(B)$ the convex hull of $B$.

\section{Characterizations by means of Chebyshev centers}

In order to prove our first theorem we need a couple of lemmas. Of course, a few pictures would be a help in understanding the meaning and the proofs of these lemmas.

To avoid trivial situations we will always suppose that the vertices of our triangles are not on a line, and we will also suppose that the dimension of the normed linear spaces involved is at least two.

LEMMA 1. Let $(X,\|\cdot\|)$ be a real normed linear space, let $\Delta=\left\{a_{1}, a_{2}, a_{3}\right\}$ be a three point subset of $X$ and suppose that $\Delta$ has a Chebyshev center $s \in X$. Then the maximum $r(s, \Delta)=\max _{1 \leq i \leq 3}\left\|a_{i}-s\right\|$ is attained at least at two points.

Proof. Write $r=r(s, \Delta)$ and suppose, for instance, that $\left\|s-a_{1}\right\|<$ $\left\|s-a_{3}\right\|=r$. We must show that $\left\|s-a_{2}\right\|=\left\|s-a_{3}\right\|=r$. If we assume $\left\|s-a_{2}\right\|<\left\|s-a_{3}\right\|=r$, by the continuity of the norm, there exists $s^{\prime} \in\left[s, a_{3}\right]$ such that

$$
\begin{aligned}
& \left\|s^{\prime}-a_{3}\right\|<\left\|s-a_{3}\right\|=r, \\
& \left\|s^{\prime}-a_{2}\right\|<\left\|s-a_{3}\right\|=r \quad \text { and } \\
& \left\|s^{\prime}-a_{1}\right\|<\left\|s-a_{3}\right\|=r .
\end{aligned}
$$

Of course, this means $r\left(s^{\prime}, \Delta\right)<r=r(s, \Delta)$, which contradicts the fact that $s$ is a Chebyshev center of $\Delta$.

The following lemma is inspired by Lemma 15.1 of [1].

Lemma 2. Let $(X,\|\cdot\|)$ be a real normed linear space and let $\Delta=$ $\left\{a_{1}, a_{2}, a_{3}\right\}$ be a three point subset of $X$ such that $Z(\Delta)$ is non-empty. Then at least one of the following holds:

(a) $\Delta=\left\{a_{1}, a_{2}, a_{3}\right\}$ has a Chebyshev center which is equidistant to the three points $a_{1}, a_{2}, a_{3}$.

(b) The triangle $\Delta=\left\{a_{1}, a_{2}, a_{3}\right\}$ has a Chebyshev center in the midpoint of one of its sides.

Proof. Assume that (a) does not hold. Take $s \in Z(\Delta)$ and write $r=$ $r(s, \Delta)$. By the preceding lemma, we may suppose, without loss of generality, that

$$
\left\|s-a_{1}\right\|<\left\|s-a_{2}\right\|=\left\|s-a_{3}\right\|=r .
$$


Our aim now is to show that $m=\frac{1}{2}\left(a_{2}+a_{3}\right)$ is a Chebyshev center of $\Delta$. This will complete the proof.

Notice first that

$$
\left\|a_{2}-a_{3}\right\| \leq\left\|a_{2}-s\right\|+\left\|s-a_{3}\right\|=2 r .
$$

Next let us show that $\left\|a_{2}-a_{3}\right\|=2 r$. Assume this is not the case. Then, since $m$ is the midpoint of the segment $\left[a_{2}, a_{3}\right]$, we have

$$
\left\|m-a_{2}\right\|=\left\|m-a_{3}\right\|<r .
$$

In other words, if we denote by $\stackrel{\circ}{B}(a, r)$ the open ball centered at $a$ with radius $r$, we have $m \in \stackrel{\circ}{B}\left(a_{2}, r\right) \cap \stackrel{\circ}{B}\left(a_{3}, r\right)$. Therefore

$$
[m, s) \subset \stackrel{\circ}{B}\left(a_{2}, r\right) \cap \stackrel{\circ}{B}\left(a_{3}, r\right) .
$$

Since $\left\|s-a_{1}\right\|<r$, there exists $\bar{s} \in[m, s)$ satisfying

$$
\left\|\bar{s}-a_{1}\right\|<r .
$$

On the other hand, we have $\bar{s} \in[m, s) \subset \stackrel{\circ}{B}\left(a_{2}, r\right) \cap \stackrel{\circ}{B}\left(a_{3}, r\right)$. Thus

$$
\left\|\bar{s}-a_{2}\right\|<r \quad \text { and } \quad\left\|\bar{s}-a_{3}\right\|<r .
$$

So we have $r(\bar{s}, \Delta)<r=r(\bar{s}, \Delta)$, which contradicts the fact that $s$ is a Chebyshev center of $\Delta$. This shows that

$$
\left\|a_{2}-a_{3}\right\|=2 r
$$

and so

$$
\left\|m-a_{2}\right\|=\left\|m-a_{3}\right\|=\frac{1}{2}\left\|a_{2}-a_{3}\right\|=r .
$$

Let us now show that $m$ is a Chebyshev center of $\Delta$. If $\left\|m-a_{1}\right\| \leq r$, this is clear. Hence we assume $\left\|m-a_{1}\right\|>r$, and try to get a contradiction.

The equality $\left\|a_{2}-a_{3}\right\|=2 r$ implies that

$$
B\left(a_{2}, r\right) \cap B\left(a_{3}, r\right) \subset\left\{x \in X:\left\|x-a_{2}\right\|=\left\|x-a_{3}\right\|=r\right\} .
$$

Therefore, since $m$ and $s$ belong to $B\left(a_{2}, r\right) \cap B\left(a_{3}, r\right)$, it follows that

$$
[m, s] \subset B\left(a_{2}, r\right) \cap B\left(a_{3}, r\right) \subset\left\{x \in X:\left\|x-a_{2}\right\|=\left\|x-a_{3}\right\|=r\right\} .
$$

The function $x \mapsto\left\|x-a_{1}\right\|$ takes at $m$, a value greater than $r\left(\left\|m-a_{1}\right\|\right)$ and at $s$, a value smaller $\left(\left\|s-a_{1}\right\|\right)$. Therefore (Bolzano's theorem) at some point $s_{0} \in[m, s]$, we have $\left\|s_{0}-a_{1}\right\|=r$. But $[m, s] \subset\left\{x \in X:\left\|x-a_{2}\right\|=\right.$ 
$\left.\left\|x-a_{3}\right\|=r\right\}$ implies that $\left\|s_{0}-a_{1}\right\|=\left\|s_{0}-a_{2}\right\|=\left\|s_{0}-a_{3}\right\|=r$. This is the desired contradiction because we are assuming that condition (a) does not hold.

We can now prove the announced result.

Theorem 3. Let $X$ be a real normed linear space of dimension at least three. Then the following are equivalent:

(*) $X$ is an IPS.

(A) If $a_{1}, a_{2}, a_{3}$ are norm one points in $X$ then $Z\left(\left\{a_{1}, a_{2}, a_{3}\right\}\right)$ is nonempty, and if $\mathbf{0}$ is a Chebyshev center of the set $\left\{a_{1}, a_{2}, a_{3}\right\}$, then $\mathbf{0}$ is in its convex hull.

$\left(\mathrm{GK}^{s}\right)$ Every three point subset of $S_{X}$ has a Chebyshev center in its convex hull.

Proof. It is clear that $(*)$ implies (A).

Let us prove that (A) implies $\left(\mathrm{GK}^{s}\right)$. Let $\Delta=\left\{a_{1}, a_{2}, a_{3}\right\}$ be a three point subset of $S_{X}$. We wish to show that $Z(\Delta) \cap \operatorname{conv}(\Delta) \neq \emptyset$. The hypothesis implies that $Z(\Delta) \neq \varnothing$ and so we must be in one of the cases (a) or (b) described in Lemma 2. If we are in case (b) then clearly $Z(\Delta) \cap \operatorname{conv}(\Delta) \neq \emptyset$. So let us suppose we are in case (a). This means that there exists $b \in Z(\Delta)$ such that

$$
r(b, \Delta)=\left\|a_{1}-b\right\|=\left\|a_{2}-b\right\|=\left\|a_{3}-b\right\| .
$$

Write $r(b, \Delta)=r$ and take

$$
u_{1}=\frac{a_{1}-b}{r}, \quad u_{2}=\frac{a_{2}-b}{r}, \quad u_{3}=\frac{a_{3}-b}{r} .
$$

Then $\Delta_{0}=\left\{u_{1}, u_{2}, u_{3}\right\}$ is a three point subset of $S_{X}$. Besides, $\Delta_{0}$ is obtained from $\Delta$ through a translation and a homothety: to be precise $\Delta_{0}=\phi(\Delta)$, where $\phi(x)=\frac{x-b}{r}$. A straightforward verification now shows that $\phi(b)=\mathbf{0} \in$ $Z\left(\Delta_{0}\right)$. Our hypothesis implies that $\phi(b)=\mathbf{0} \in \operatorname{conv}\left(\Delta_{0}\right)=\operatorname{conv}(\phi(\Delta))$. Of course, it follows that $b \in \operatorname{conv}(\Delta)$. Hence we get $Z(\Delta) \cap \operatorname{conv}(\Delta) \neq \emptyset$, as we wished.

Finally, let us prove that $\left(\mathrm{GK}^{s}\right)$ implies (*). Let us suppose that $X$ is not an IPS. By the Garkavi-Klee theorem, this means that (GK) does not hold. So we can find a three point subset $\Delta=\left\{a_{1}, a_{2}, a_{3}\right\}$ of $X$ such that $Z(\Delta) \cap \operatorname{conv}(\Delta)=$ $\emptyset$. Therefore, there exists $y_{0} \in X \backslash \operatorname{conv}(\Delta)$, such that

$$
r\left(y_{0}, \Delta\right)=\max _{1 \leq i \leq 3}\left\|a_{i}-y_{0}\right\|<r(x, \Delta) \quad \text { for all } \quad x \in \operatorname{conv}(\Delta) .
$$


Let us denote by $Y$ the linear span of $\left\{a_{1}, a_{2}, a_{3}, y_{0}\right\}$, and let $b \in Y$ be a Chebyshev center of $\Delta=\left\{a_{1}, a_{2}, a_{3}\right\}$ in the finite dimensional space $Y$,

$$
r(b, \Delta)=\max _{1 \leq i \leq 3}\left\|a_{i}-b\right\| \leq r(y, \Delta) \quad \text { for all } \quad y \in Y .
$$

In particular, taking $y=y_{0}$, we deduce $r(b, \Delta) \leq r\left(y_{0}, \Delta\right)<r(x, \Delta)$ for all $x \in \operatorname{conv}(\Delta)$, and so

$$
r(b, \Delta)<r(x, \Delta) \quad \text { for all } \quad x \in \operatorname{conv}(\Delta) .
$$

This inequality implies $b \notin \operatorname{conv}(\Delta)$. Therefore, condition (b) in Lemma 2 can not be satisfied, and so condition (a) in that lemma holds. Hence, we can assume

$$
r(b, \Delta)=\left\|a_{1}-b\right\|=\left\|a_{2}-b\right\|=\left\|a_{3}-b\right\| \leq r(y, \Delta) \quad \text { for all } \quad y \in Y .
$$

We proceed now as in the previous reasoning. Write $r(b, \Delta)=r$ and take $u_{1}=\frac{a_{1}-b}{r}, u_{2}=\frac{a_{2}-b}{r}$ and $u_{3}=\frac{a_{3}-b}{r}$. Then $\Delta_{0}=\left\{u_{1}, u_{2}, u_{3}\right\}$ is a three point subset of $S_{X}$. As above we have $\Delta_{0}=\phi(\Delta)$, where $\phi(x)=\frac{x-b}{r}$. So, by a straightforward verification, we get from (1)

$$
r\left(\mathbf{0}, \Delta_{0}\right)=r(\phi(b), \phi(\Delta))<r(\phi(x), \phi(\Delta)) \quad \text { for all } \quad x \in \operatorname{conv}(\Delta),
$$

and so

$$
r\left(\mathbf{0}, \Delta_{0}\right)<r\left(z, \Delta_{0}\right) \quad \text { for all } \quad z \in \operatorname{conv}\left(\Delta_{0}\right) .
$$

Of course, this implies that $\Delta_{0}$ has no Chebyshev center in its convex hull, and this means that $\left(\mathrm{GK}^{s}\right)$ does not hold.

REMARK 2. In the preceding proof it was shown that for all real normed linear spaces (whatever their dimension) conditions $\left(\mathrm{GK}_{\infty}^{s}\right)$ and $\left(\mathrm{GK}_{\infty}\right)$ are equivalent.

\section{Characterizations by means of Fermat centers}

To get the characterizations we need two results. The first one is due to Durier. The second one is a consequence of Benítez-Fernández-Soriano theorem (Theorem 2 above) in the case $p=1$.

Proposition 1 (Corollary 2.3 of [5]). Let $X$ be a real normed linear space, let $\Delta=\left\{a_{1}, a_{2}, a_{3}\right\}$ be a three point subset of $X$, let $\lambda_{1}, \lambda_{2}, \lambda_{3}$ be positive numbers and consider the three point set $\Delta^{\prime}=\left\{\lambda_{1} a_{1}, \lambda_{2} a_{2}, \lambda_{3} a_{3}\right\}$. Suppose $\mathbf{0} \in Z^{1}(\Delta)$. Then the following is true:

(1) $\mathbf{0} \in Z^{1}\left(\Delta^{\prime}\right)$. 
(2) If $\lambda_{i} \leq 1$ for $i=1,2,3$ then $Z^{1}\left(\Delta^{\prime}\right) \subset Z^{1}(\Delta)$.

(3) If $\lambda_{i}=\lambda$ for $i=1,2,3$ then $Z^{1}\left(\Delta^{\prime}\right)=\lambda Z^{1}(\Delta)$.

Let $X$ be a real normed linear space, and let $Y$ be a subspace of $X$. Given a three point subset $\Delta=\left\{a_{1}, a_{2}, a_{3}\right\}$ of $Y$, we denote by $Z_{Y}^{1}(\Delta)$ the set of all Fermat centers of $\Delta$ in $Y$, that is,

$$
Z_{Y}^{1}(\Delta)=\left\{z \in Y: r_{1}(z, \Delta)=\inf _{y \in Y} r_{1}(y, \Delta)\right\} .
$$

Lemma 3. Let $X$ be a real normed linear space of dimension at least three, and let us suppose $X$ is not an IPS. Then there exist a subspace $Y$ of $X$ and a three point subset $\Delta$ of $S_{Y}$ such that $\mathbf{0} \in Z_{Y}^{1}(\Delta)$ and $Z_{Y}^{1}(\Delta) \cap \operatorname{conv}(\Delta)=\emptyset$.

Proof. Assume $X$ is not an IPS. By the Benítez-Fernández-Soriano theorem, there is a three point subset $T=\left\{a_{1}, a_{2}, a_{3}\right\}$ of $X$ such that $Z^{1}(T) \cap$ $\operatorname{conv}(T)=\emptyset$. Therefore, there exists $y_{0} \in X$ such that

$$
r_{1}\left(y_{0}, T\right)<r_{1}(x, T) \quad \text { for all } \quad x \in \operatorname{conv}(T) .
$$

Then, if we denote by $Y$ the linear span of $\left\{a_{1}, a_{2}, a_{3}, y_{0}\right\}$, we have

$$
Z_{Y}^{1}(T) \cap \operatorname{conv}(T)=\emptyset .
$$

If we apply the Hahn-Banach separation theorem on $Y$ to the compact convex sets $Z_{Y}^{1}(T)$ and $\operatorname{conv}(T)$, we deduce that there exist a linear form $y^{*}$ on $Y$ and some real number $c$ such that

$$
y^{*}(y) \leq c<y^{*}(x) \quad \text { for all } y \in Z_{Y}^{1}(T) \text { and all } x \in \operatorname{conv}(T) .
$$

Of course, we can assume there exists $b \in Z_{Y}^{1}(T)$ such that $y^{*}(b)=c$. Moreover, by means of the translation $x \mapsto x-b$, we can suppose that $b=\mathbf{0}$ and $c=0$ so that

$$
y^{*}(y) \leq 0<y^{*}(x) \quad \text { for all } y \in Z_{Y}^{1}(T) \text { and all } x \in \operatorname{conv}(T) .
$$

Notice now that the preceding inequality implies that $a_{i} \neq \mathbf{0}$ for $i=1,2,3$. Take $\lambda=\min \left\{\left\|a_{1}\right\|,\left\|a_{2}\right\|,\left\|a_{3}\right\|\right\}$, and take $a_{i}^{\prime}=\frac{1}{\lambda} a_{i}$ for $i=1,2,3$. By parts 1 and 3 of the preceding proposition, if we write $T^{\prime}=\left\{a_{1}^{\prime}, a_{2}^{\prime}, a_{3}^{\prime}\right\}$, we have $\mathbf{0} \in Z_{Y}^{1}\left(T^{\prime}\right)$ and

$$
y^{*}(y) \leq 0<y^{*}(x) \quad \text { for all } y \in Z_{Y}^{1}\left(T^{\prime}\right) \text { and all } x \in \operatorname{conv}\left(T^{\prime}\right) .
$$

Take

$$
\Delta=\left\{\frac{a_{1}^{\prime}}{\left\|a_{1}^{\prime}\right\|}, \frac{a_{2}^{\prime}}{\left\|a_{2}^{\prime}\right\|}, \frac{a_{3}^{\prime}}{\left\|a_{3}^{\prime}\right\|}\right\} .
$$


Since $\left\|a_{i}^{\prime}\right\| \geq 1$ for $i=1,2,3$, it follows from parts 1 and 2 of the preceding proposition that $\mathbf{0} \in Z_{Y}^{1}(\Delta)$ and

$$
y^{*}(y) \leq 0<y^{*}(x) \quad \text { for all } y \in Z_{Y}^{1}(\Delta) \text { and all } x \in \operatorname{conv}(\Delta) .
$$

Of course, $\Delta$ is a three point subset of $S_{X}$ and the preceding inequality implies that $Z_{Y}^{1}(\Delta)$ and $\operatorname{conv}(\Delta)$ are disjoint. This completes the proof.

We can now give our second characterization of inner product spaces.

THEOREM 4. Let $X$ be a real normed linear space of dimension at least three. Then the following are equivalent:

(*) $X$ is an IPS.

$\left(\mathrm{A}_{1}\right)$ If $a_{1}, a_{2}, a_{3}$ are norm one points in $X$ then $Z^{1}\left(\left\{a_{1}, a_{2}, a_{3}\right\}\right)$ is nonempty, and if $\mathbf{0}$ is a Fermat center of the set $\left\{a_{1}, a_{2}, a_{3}\right\}$, then $\mathbf{0}$ is in its convex hull.

$\left(\mathrm{GK}_{1}^{s}\right)$ Every three point subset of $S_{X}$ has a Fermat center in its convex hull.

Proof. It is clear that $(*)$ implies $\left(\mathrm{A}_{1}\right)$.

Let us prove that $\left(\mathrm{A}_{1}\right)$ implies $\left(\mathrm{GK}_{1}^{s}\right)$. Let $T=\left\{a_{1}, a_{2}, a_{3}\right\}$ be a three point subset of $S_{X}$. We must prove that $Z^{1}(T) \cap \operatorname{conv}(T) \neq \emptyset$. By our hypothesis, $Z^{1}(T) \neq \emptyset$. Take $b \in Z^{1}(T)$ (we will assume $b \neq a_{i}$ for $i=1,2$, 3, otherwise we would trivially have $Z^{1}(T) \cap \operatorname{conv}(T) \neq \emptyset$ ). Then $\mathbf{0} \in Z^{1}(\Delta)$, where $\Delta=\left\{a_{1}-b, a_{2}-b, a_{3}-b\right\}$. Take $\lambda=\min \left\{\left\|a_{1}-b\right\|,\left\|a_{2}-b\right\|,\left\|a_{3}-b\right\|\right\}$. Since $\lambda$ is a positive number, we can define $a_{i}^{\prime}=\frac{1}{\lambda}\left(a_{i}-b\right)$, for $i=1,2,3$. By part 1 of Proposition $1, \mathbf{0} \in Z^{1}\left(\Delta^{\prime}\right)$, where $\Delta^{\prime}=\left\{a_{1}^{\prime}, a_{2}^{\prime}, a_{3}^{\prime}\right\}$. Notice that we now have $\left\|a_{i}^{\prime}\right\| \geq 1$ for $i=1,2,3$. Therefore, it follows from parts 1 and 2 of Proposition 1 that

$$
\mathbf{0} \in Z^{1}\left(\Delta^{\prime \prime}\right) \subset Z^{1}\left(\Delta^{\prime}\right)
$$

where

$$
\Delta^{\prime \prime}=\left\{\frac{a_{1}^{\prime}}{\left\|a_{1}^{\prime}\right\|}, \frac{a_{2}^{\prime}}{\left\|a_{2}^{\prime}\right\|}, \frac{a_{3}^{\prime}}{\left\|a_{3}^{\prime}\right\|}\right\} .
$$

Now, the hypothesis implies that $\mathbf{0}$ belongs to $\operatorname{conv}\left(\Delta^{\prime \prime}\right)$. Using this one can easily verify that $b$ belongs to $\operatorname{conv}(T)$. This completes the proof of this part.

Finally, let us show that $\left(\mathrm{GK}_{1}^{s}\right)$ implies $(*)$. Assume $(*)$ does not hold, that is, assume $X$ is not an IPS. By the preceding lemma, there exist a subspace $Y$ of $X$ and a three point subset $\Delta$ of $S_{Y}$ such that $\mathbf{0} \in Z_{Y}^{1}(\Delta)$ and $Z_{Y}^{1}(\Delta) \cap \operatorname{conv}(\Delta)=$ $\emptyset$. Therefore,

$$
\min \left\{r_{1}(y, \Delta): y \in Y\right\}<\min \left\{r_{1}(x, \Delta): x \in \operatorname{conv}(\Delta)\right\} .
$$


But this implies

$$
\begin{aligned}
\inf \left\{r_{1}(x, \Delta): x \in X\right\} & \leq \min \left\{r_{1}(y, \Delta): y \in Y\right\} \\
& <\min \left\{r_{1}(x, \Delta): x \in \operatorname{conv}(\Delta)\right\} .
\end{aligned}
$$

It then follows that $Z^{1}(\Delta)$ and $\operatorname{conv}(\Delta)$ are disjoints, and this completes the proof.

\section{A characterization by means of $p$-centers}

The following proposition is, at least partially, known (see Lemma 5.2 of [5], and also [3] and [4]). However, we have not found the whole statement of the proposition explicitly in the literature. For this reason we include a proof. It relies upon results contained in [3] and [4].

Proposition 2. Let $X$ be a real normed linear space, let $a_{1}, a_{2}, a_{3}$ be norm one points in $X$ and let $p>1$. Then $\mathbf{0} \in Z^{p}(\Delta)$ if and only if $\mathbf{0} \in Z^{1}(\Delta)$, and in this case $Z^{p}(\Delta) \subset Z^{1}(\Delta)$.

Proof. We use the notations of [3] and [4]. By Lemma 1 of [3], $0 \in Z^{p}(\Delta)$ if and only if there exist $f \in J a_{1}, g \in J a_{2}, h \in J a_{3}$, such that

$$
\left\|a_{1}\right\|^{p-1} f+\left\|a_{2}\right\|^{p-1} g+\left\|a_{3}\right\|^{p-1} h=f+g+h=0 .
$$

But by Proposition 1 of [4], this just means that $\mathbf{0} \in Z^{1}(\Delta)$.

Let us show now that $Z^{p}(\Delta) \subset Z^{1}(\Delta)$. Take $x \in Z^{p}(\Delta)$. Lemma 2 of [3] implies that

$$
\left\|a_{i}\right\|=\left\|a_{i}-x\right\| \quad \text { for all } \quad i \in\{1,2,3\},
$$

and so

$r_{1}(\mathbf{0}, \Delta)=\left\|a_{1}\right\|+\left\|a_{2}\right\|+\left\|a_{3}\right\|=\left\|a_{1}-x\right\|+\left\|a_{2}-x\right\|+\left\|a_{3}-x\right\|=r_{1}(x, \Delta)$.

Therefore, $x \in Z^{1}(\Delta)$.

We can give now the characterization of inner product spaces. It is a consequence of the preceding proposition and Lemma 3.

THEOREM 5. Let $X$ be a real normed linear space of dimension at least three, and let $p>1$. Then the following are equivalent:

(*) $X$ is an IPS.

$\left(\mathrm{GK}_{p}^{s}\right)$ Every three point subset of $S_{X}$ has a p-center in its convex hull.

Proof. It is well known that $(*)$ implies $\left(\mathrm{GK}_{p}^{s}\right)$. For the converse, assume $X$ is not an IPS. By Lemma 3, there exist a subspace $Y$ of $X$ and a three point 
subset $\Delta$ of $S_{Y}$ such that $\mathbf{0} \in Z_{Y}^{1}(\Delta)$ and $Z_{Y}^{1}(\Delta) \cap \operatorname{conv}(\Delta)=\emptyset$. Then, by the preceding proposition, $Z_{Y}^{p}(\Delta) \cap \operatorname{conv}(\Delta)=\emptyset$. Therefore,

$$
\min \left\{r_{p}(y, \Delta): y \in Y\right\}<\min \left\{r_{p}(x, \Delta): x \in \operatorname{conv}(\Delta)\right\} .
$$

But this implies

$$
\begin{aligned}
\inf \left\{r_{p}(x, \Delta): x \in X\right\} & \leq \min \left\{r_{p}(y, \Delta): y \in Y\right\} \\
& <\min \left\{r_{p}(x, \Delta): x \in \operatorname{conv}(\Delta)\right\} .
\end{aligned}
$$

It follows that $Z^{p}(\Delta)$ and $\operatorname{conv}(\Delta)$ are disjoint, and this finishes the proof.

ACKNOWLEDGEMENTS. We are very grateful to the referees for useful comments and suggestions which have led us to improve very much the readability of the paper.

\section{REFERENCES}

1. Amir, D., Characterizations of Inner Product Spaces, Birkhäuser Verlag, Basel, 1986.

2. Benítez, C., Fernández, M., and Soriano, L., Location of the 2-centers of three points, Rev. R. Acad. Cienc. Exact. Fís. Natur. Madrid (Esp.) 94 (2000), 515-517.

3. Benítez, C., Fernández, M., and Soriano, L., Weighted p-Centers and the convex hull property, Numer. Funct. Anal. Optim. 23 (2002), 39-45.

4. Benítez, C., Fernández, M., and Soriano, L., Location of the Fermat-Torricelli medians of three points, Trans. Amer. Math. Soc. 304 (2002), 5027-5038.

5. Durier, R., Optimal locations and inner products, J. Math. Anal. Appl. 207 (1997), 220-239.

6. Garkavi, A. L., On the Chebyshev center and the convex hull of a set, Uspekhi Mat. Nauk USSR 19 (1964), 139-145.

7. Klee, V., Circumspheres and inner products, Math. Scand. 8 (1960), 363-370.

8. Mendoza, J., and Pakhrou, T., On some characterizations of inner product spaces, J. Math. Anal. Appl. 282 (2003), 369-382.

DEPARTAMENTO DE ANÁLISIS MATEMÁTICO

UNIVERSIDAD COMPLUTENSE DE MADRID

28040 MADRID

SPAIN

E-mail: Jose_Mendoza@mat.ucm.es, Tijani_Pakhrou@mat.ucm.es 